\title{
APLICAÇÃO DA EQUAÇÃO UNIVERSAL DE PERDAS DE SOLO NA REGIÃO SUL DO AMAZONAS
}

Juliana Gervasio NUNES ${ }^{1}$

Milton César Costa CAMPOS ${ }^{2}$

Junior Cesar NUNES ${ }^{3}$

Bruno Campos MANTOVANELLI ${ }^{4}$

Jose Mauricio da CUNHA

Marcelo Dayron Rodrigues SOARES ${ }^{6}$

\begin{abstract}
${ }^{1}$ Engenheira Ambiental, Professora do Instituto de Ciência e Tecnologia - IFMT, campus Sorriso-MT. e-mail: jugervasionunes@hotmail.com.

${ }^{2}$ Engenheiro Agrônomo, Professor Dr. Adjunto IV, Bolsista de Produtividade do CNPq - Universidade Federal do Amazonas - UFAM - Campus de Humaitá, e-mail: mcesarsolos@ gmail.com.

${ }^{3}$ Engenheiro Agrônomo, Instituto de Educação, Agricultura e Ambiente, Universidade Federal do Amazonas. E-mail: jugervasionunes@hotmail.com.

${ }^{4}$ Engenheiro Agrônomo, Mestrando pelo Programa de Pós Graduação em Ciência do Solo da Universidade Federal Rural de Pernambuco - UFRPE - Recife, e-mail: brunomantovanelli21 @ gmail.com.

${ }^{5}$ Bacharel em Física, Professor Doutorando do Programa de Pós Graduação em Física Ambiental Adjunto I Universidade Federal do Amazonas - UFAM - Campus de Humaitá, e-mail: maujmc@gmail.com.

6Engenheiro Ambiental, Professor do Instituto de Educação Agricultura e Ambiente - IEAA, UFAM - Campus Humaitá. e-mail: marcelo.dayron@gmail.com.
\end{abstract}

Recebido em: 14/05/2016 - Aprovado em: 14/11/2017 - Disponibilizado em: 30/12/2017

\section{RESUMO:}

O objetivo deste trabalho foi aplicar a Equação Universal de Perdas de Solo (USLE), tendo como referência a estimativa da tolerância de perdas de solo para as principais ordens de solo na região Sul do Estado do Amazonas.As perdas potenciais de solo variaram de 149,72 (Argissolos) a 25,41 $\mathrm{t} \mathrm{ha}^{-1}$ ano $^{-1}$ (Gleissolos) para o solo desnudo e plantio morro abaixo, de 0,96 (Argissolos) a $0,16 \mathrm{t} \mathrm{ha}^{-1}$ ano $^{-1}$ (Gleissolos) para a combinação de pastagem e semeadura em contorno, e de 18,97(Argissolos) a 3,22 $\mathrm{t} \mathrm{ha}^{-1}$ ano $^{-1}$ (Gleissolos) para a combinação de cultura do milho com semeadura em contorno. $\mathrm{O}$ uso de valores de tolerância em modelos de predição de perdas de solo permitiu buscar a combinação de fatores $\mathrm{C}$ (manejo) e $\mathrm{P}$ (prática) que promova uma perda de solos menores que o limite tolerável.

Palavras chave: Perda de solo, Limites de tolerância, Amazonas.

\section{SOIL LOSS TOLERANCE IN SOUTHERN AMAZON}

\begin{abstract}
:
The objective of this study was to apply the Universal Soil Loss Equation (USLE), with reference to the estimated soil loss tolerance for the major soil orders in the southern state of Amazonas. As potential loss of soil region ranged from 14972 (Argissolos) to $25.41 \mathrm{t} \mathrm{ha}^{-1} \mathrm{yr}-1$ (Gleissolo) for bare soil and planting hill below 0.96 (Argissolos) to $0.16 \mathrm{t} \mathrm{ha}^{-1}$ year $^{-1}$ (Gleissolo) to the combination of pasture and seeding in contour, and 18.97 (Argissolo) to $3.22 \mathrm{t}^{\mathrm{ha}-1} \mathrm{year}^{-1}$ (Gleissolo) for combining corn crop with sowing in contour. The use of tolerance values in soil loss prediction models allowed to seek a combination of factors $\mathrm{C}$ (management) and $\mathrm{P}$ (practice) that promotes a loss of smaller soil the tolerable limit.
\end{abstract}

Keywords: Soil Loss Tolerance limits, Amazonas. 


\section{Introdução}

A perda de solos por erosão hídrica constitui-se em um sério problema devido, fundamentalmente, à substituição da vegetação natural capaz de recobrir e proteger o solo do que as culturas em geral implantadas pelo homem. Tal fato não impede que existam solos que, devido à tolerância natural à perda, comportem distintas coberturas menos protetoras, sem apresentar diminuição apreciável na sua produtividade ao longo do tempo (GERARDO, 2011).

O uso de modelos de predição para avaliar as perdas de material de uma área cultivada vem se tornando prática indispensável para o planejamento conservacionista (OLIVEIRA, 2004). A equação universal de perdas de solo (EUPS), desenvolvida por pesquisadores do "Agricultural Research Service” (ARS), vem sendo aplicada a mais de 30 anos, como um método para a predição das perdas médias anuais de solo por erosão (MARTINS FILHO, 2004).

$$
\text { Wischmeier e Smith }
$$
propuseram a equação universal de perdas de solo (EUPS), na qual os cinco fatores que levam em consideração os fatores relacionados à erosão são expressos. No entanto a EUPS recebe críticas quanto a estas finalidades ou aplicações por não poder ser aplicada em estimativas de perdas de solo para um ano ou evento específico. Entretanto, mesmo com suas limitações, a EUPS tem sido empregada como um guia para os profissionais da área de conservação do solo no planejamento dos cultivos, manejos e práticas de conservação (MARTINS FILHO, 2004). Segundo Oliveira (2004) a equação é muito utilizada no Brasil, servindo para quantificar a erosão e tem o objetivo principal servir de guia para o uso mais racional da terra.

Na Região Sul do Amazonas (região do Médio Madeira) há uma tendência crescente de avanço da agricultura, sobretudo da pecuária, e com isso há um crescimento das áreas de pastagem. Entretanto os tecnologias, tanto para uso do solo quanto para utilização de práticas conservacionistas mecânicas, edáficas e vegetativas que atuem no controle da erosão e recuperação de áreas degradadas, são muito baixos e demandam primeiro por uma estimação de perdas de solo que permita guiar a escolha de atividades a serem.

Este trabalho tem como objetivos a avaliação das perdas de solos através da aplicação da Equação Universal de Perdas de Solo (Wischmeier Smith, 1978) para as Ordens de solos representativas da região Sul do estado do Amazonas a partir da estimativa da tolerância a perdas de solo a fim de propor uma ferramenta que ajude a delinear alternativas compatíveis com os limites de tolerância intrínseca de cada classe de solo presente na região de estudo,de forma a melhor planejar o uso e manejo do solo garantindo a sustentabilidade das áreas produtivas. 


\section{Materiais e Métodos}

O estudo foi realizado no Instituto de Educação, Agricultura e Ambiente da Universidade Federal do Amazonas-UFAM, a partir da estimativa da tolerância de perdas de solo para as principais Ordens de solos da região Sul do Amazonas. Os valores médios estimados foram utilizados para realizar a simulação com a aplicação da USLE para diversas condições.

Os valores de tolerância de perdas de solo foram estimadas através do método de Galindo e Margolis (1989), que considera além da profundidade efetiva e relação textural a introdução do teor de argila do horizonte A como variável associada à relação textural que resultaram em novos valores para a variável $r_{a}$.

$\mathrm{T}=\mathrm{h} \cdot \mathrm{r}_{\mathrm{a}} \cdot \mathrm{m} \cdot \mathrm{p} \cdot 1.000^{-1}$

Onde: $\mathrm{T}=$ tolerância de perda de solo ( $\mathrm{t}$ ha-1 ano-1); $\mathrm{h}=$ profundidade efetiva do solo $(\mathrm{mm})$, limitada a $1.000 \mathrm{~mm} ; r_{a}=$ relação que expressa, conjuntamente, o efeito da relação textural entre os horizontes $\mathrm{B}$ ou $\mathrm{C}$ e $\mathrm{A}$ e do teor de argila do horizonte $\mathrm{A} ; 1.000=$ constante que expressa o período de tempo para haver o desgaste de uma camada de solo de $1.000 \mathrm{~mm}(1 \mathrm{~m})$ de espessura, desconsiderando a formação de solo nesse período, segundo o procedimento de Lombardi Neto e Bertoni (1975); $\mathrm{m}$ = fator que expressa o efeito da matéria orgânica na camada de 0 $20 \mathrm{~cm} ; \mathrm{p}=$ fator que expressa o efeito da permeabilidade do solo.
Com referência ao teor de matéria orgânica dos solos, estabeleceu-se o critério de Galindo \& Margolis (1989) para o cálculo da perda aceitável de solo: (a) para solos com teor de matéria orgânica maior que $2 \%$, multiplicou-se a espessura da camada de solo calculada pelo fator 1,15; (b) para teores de matéria orgânica entre 1 e $2 \%$, multiplicou-se a espessura da camada de solo calculada pelo fator 1,00; (c) para solos com teor de matéria orgânica menor que $1 \%$, multiplicou-se a espessura da camada de solo calculada pelo fator 0,85 .

A permeabilidade de cada horizonte dos perfis estudados baseou-se em informações de textura e grau de desenvolvimento da estrutura do solo nos respectivos horizontes obtidos no banco de dados, segundo metodologia de Galindo e Margolis (1989): (a) para uma permeabilidade rápida, multiplicou-se a espessura da camada de solo calculada pelo fator 1,15; (b) para uma permeabilidade moderada, multiplicou-se a espessura da camada de solo calculada pelo fator 1,00; e (c) para uma permeabilidade lenta, multiplicou-se a espessura da camada de solo calculada pelo fator 0,85. Quando encontrado uma estrutura maciça coesa esta foi considerada como forte para termos de permeabilidade.

Para o cálculo da EUPS o fator de erosividade $(\mathrm{R})$ utilizado é de valor igual a 14129 MJ mm ha ${ }^{-1} \mathrm{~h}^{-1}$ ano $^{-1}$ que correspondente a Manaus, capital do 
Amazonas (MARTINS FILHO, 2004).

Para a determinação do fator $\mathrm{K}$ a equação (1) utilizada foi a de Denardin (1990), e para o fator LS a equação (2) estabelecida por Bertoni (1959):

(1) $\mathrm{K}=0,0061 \mathrm{P}+0,0083 \mathrm{MO}-0,0012 \mathrm{Al}-$ 0,0004 PART; (2) LS $=0,00984 \mathrm{C}^{0,63} . \mathrm{D}^{1,18}$

Os solos estudados foram submetidos a três condições simuladas distintas de manejo para fins de comparação das perdas com os limites toleráveis. Os fatores $\mathrm{C}$ utilizados foram solo desnudo $(1,0000)$, cultura do milho (0,1980), e pastagem $(0,0100)($ Roose, 1977) e correspondem respectivamente a fatores $\mathrm{P}$ de plantio morro à abaixo $(1,00)$, semeadura em contorno $(0,64)$ e, novamente, semeadura em contorno $(0,64)$. Os valores de tolerância de perdas estimadas para cada classe de solo foram utilizados em conjunto com a EUPS, considerando-se um valor médio para cada Ordem de solo, visando obter resultados que contribuam para o estabelecimento do uso sustentável da terra pela seleção de práticas adequadas de manejo e conservação dos solos. Os fatores $\mathrm{P}$ e $\mathrm{C}$ utilizados foram aqueles determinados por Margolis et al. (1985) e Roose (1977).

Tabela 1 - Determinação das classes de permeabilidade em função da textura e estrutura segundo Galindo e Margolis (1989).

\begin{tabular}{ccc}
\hline Textura & Estrutura & Classe de Permeabilidade \\
\hline Fina & Fraca & Lenta \\
$($ argila $\geq 35 \%)$ & Moderada & Lenta \\
& Forte & Moderada \\
Média & Fraca & Moderada \\
$(15 \% \leq$ argila $\leq 35 \%)$ & Moderada & Moderada \\
& Forte & Rápida \\
Grossa & Fraca & Moderada \\
$($ areia e areia fina $)$ & Moderada & Rápida \\
& Forte & Rápida \\
\hline
\end{tabular}

\section{Resultados e Discussão}

Foram calculados as perdas de solo e o limite de tolerância a estas perdas. Obtendo-se os valores de perda de solo e também a tolerância a essas perdas, foi possível predizer a partir da subtração dos respectivos valores que as perdas podem ser superiores ou inferiores ao limite de tolerância. Valores negativos indicam uma perda maior que o tolerável e que resulta em uma degradação do solo.

Como uma variação de 7,15 para Planossolos a $14,14 \mathrm{t} \mathrm{ha}^{-1}$ ano $^{-1}$ para Gleissolos os valores de tolerância situam-se dentro das faixas de 4,5 a 13,4 $\mathrm{t} \mathrm{ha}^{-1}$ ano-1 para solos com B textural e de 9,6 a 15,0 tha $^{-1}$ ano $^{-1}$ para solos com B latossólico encontradas por Lombardi Neto e Bertoni (1975), em estudo de tolerância 
de perdas de terra para solos do Estado de São Paulo. Para solos da região Amazônica, Vieira e Vieira (1990) obtiveram valores de tolerância de 7,8 a 9,3 $\mathrm{t} \mathrm{ha}^{-1}$ ano $^{-1}$ para solos com B textural e de 10,4 a 16,8 $\mathrm{t} \mathrm{ha}^{-1} \mathrm{ano}^{-1}$ para solos com B latossólico.

Tabela 2 - Valores médios de profundidade efetiva dos perfis, relação textural entre os horizontes B e A, e tolerância de perdas de solo por erosão para as principais Ordens da Região Sul do Amazonas.

\begin{tabular}{lcccc}
\hline Classe de solo & $\begin{array}{c}\text { Número de } \\
\text { perfis }\end{array}$ & $\begin{array}{c}\text { Profundidade } \\
\text { efetiva }\end{array}$ & $\begin{array}{c}\text { Relação } \\
\text { Textural }\end{array}$ & Tolerância a perda \\
\cline { 2 - 5 } & & $\mathrm{m}$ & $\left(\mathrm{g} \mathrm{kg}^{-1}\right)\left(\mathrm{g} \mathrm{kg}^{-1)-1}\right.$ & $\mathrm{tha}^{-1} \mathrm{ano}^{-1}$ \\
\hline Argissolo & 10 & 1,40 & & \\
Cambissolo & 12 & 1,32 & 1,93 & $8,61 \mathrm{a}$ \\
Gleissolo & 2 & 1,09 & 1,21 & $13,65 \mathrm{a}$ \\
Latossolo & 10 & 1,52 & 1,05 & $14,14 \mathrm{a}$ \\
Neossolo & 2 & 1,83 & 1,20 & $12,73 \mathrm{a}$ \\
Planossolo & 1 & 1,30 & 1,16 & $10,48 \mathrm{a}$ \\
\hline
\end{tabular}

As perdas por erosão para as ordens estudadas da região Sul do Amazonas foram calculadas de acordo com a USLE (WISCHMEIER E SMITH, 1978). Das distintas simulações de manejo a que os solos estudados foram submetidos, as maiores perdas se deram na combinação de solo desnudo e plantio morro abaixo, com uma amplitude de 149,72 (Argissolo) a 25,41 t ha ${ }^{-1}$ ano $^{-1}$ (Gleissolos), seguida pela combinação de cultura do milho e semeadura em contorno, 18,97 (Argissolo) a 3,22 $\mathrm{t} \mathrm{ha}^{-1} \mathrm{ano}^{-1}$ (Gleissolo), e pela combinação de pastagem e semeadura em contorno, 0,96 (Argissolos) a $0,16 \mathrm{t} \mathrm{ha}^{-1}$ ano-1 (Gleissolo). Esta ultima simulação obteve valores muito reduzidos em relação às demais.

Oliveira (2004) sugere que quando a previsão de perdas é superior ao limite máximo tolerado, há como buscar combinações de manejo e cultivo a serem adotadas, nas quais a predição de perdas de solo idealmente resulte menor que a tolerância proporcionando, portanto, uma condição satisfatória do controle da erosão, em termos de sustentabilidade.

As perdas potenciais de solo foram proporcionais ao limite de tolerância dos solos. Solos menos tolerantes e perderam mais e mais tolerantes perderam menos solo. A exceção se nos Argissolos. O Planossolo apesar de menos tolerante que os Argissolos perdem menos solos do que estes. Isso ocorre pela influencia dos fatores $\mathrm{K}$ e LS, respectivamente erodibilidade e comprimento e grau do declive, já que os Planossolos possuem valores de $\mathrm{K}$ e de LS menores que os Argissolos (Tabela 4).

Quando o solo desnudo (C) foi submetido a condição de plantio morro abaixo 
(P) as perdas potenciais foram mais acentuadas e muito ultrapassaram o limite de tolerância, ocorrendo nos Argissolos as perdas que mais ultrapassam os limites toleráveis, e nos Gleissolos as que menos ultrapassam.

Quando C passa a ser um cultivo de milho e P uma semeadura em contorno a comparação entre perdas e limite apresentou valor negativo apenas para os Argissolos. Ao submeter o solo ao cultivo de pastagem (C) combinada prática conservacionista de semeadura em contorno (P) os valores de perda se apresentaram positivos, ou seja, as perdas de solo ficaram abaixo do limite tolerável para todas as ordens de solo estudadas.

O cultivo de pastagem possui uma cobertura vegetal mais propícia a redução do impacto das gotas de chuva, além de um sistema radicular capaz de interferir no movimento da água no solo, que minimizam a erosão. Contudo o manejo inadequado do rebanho bovino faz com que mesmo sob pastagem existam áreas degradadas por erosão. Bertoni e Lombardi Neto (2008) afirmam que as gramíneas são adaptadas ao controle de erosão pela capacidade que possuem de diminuir a intensidade de enxurrada e prender as partículas de solo contra a pressão da água, formando pequenas rugosidades no terreno retardam o movimento da água.

Convém ressaltar que, embora não seja considerada no cálculo de tolerância, a formação de solo durante o período de tempo necessário para a remoção de uma camada de 1 metro ocorre a taxas variáveis para cada solo. Portanto, mesmo quando baixa, essa formação de solo poderá diminuir o balanço negativo dos valores de (T-A) em relação aos obtidos (OLIVEIRA, 2004). 


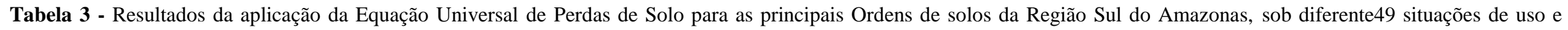
manejo.

\begin{tabular}{|c|c|c|c|c|c|c|c|c|}
\hline \multirow[t]{2}{*}{ Classes de Solos } & \multicolumn{5}{|c|}{ Equação Universal de Perda de Solo } & \multicolumn{3}{|c|}{ Tolerância } \\
\hline & $\mathrm{R}^{(1)}$ & $\mathrm{K}^{(2)}$ & $\mathrm{LS}^{(3)}$ & $\mathrm{C}^{(4)}$ & $\mathrm{P}^{(4)}$ & A & $\mathrm{T}$ & $\mathrm{T}-\mathrm{A}$ \\
\hline & MJ mm ha ${ }^{-1} \mathrm{~h}^{-1} \mathrm{ano}^{-1}$ & $\mathrm{th} \mathrm{MJ}^{-1} \mathrm{~mm}^{-1}$ & $(\mathrm{~m})(\%)$ & & & $\mathrm{tha}^{-1} \mathrm{ano}^{-1}$ & $\mathrm{tha}^{-1} \mathrm{ano}^{-1}$ & $\mathrm{t} \mathrm{ha}^{-1}$ ano $^{-1}$ \\
\hline \multirow[t]{3}{*}{ Argissolo } & 14129 & 0,0521 & 0,2034 & 1,0000 & 1,00 & 149,72 & 8,61 & $-141,11$ \\
\hline & & & & 0,1980 & 0,64 & 18,97 & & $-10,36$ \\
\hline & & & & 0,0100 & 0,64 & 0,96 & & 7,65 \\
\hline \multirow[t]{3}{*}{ Cambissolo } & 14129 & 0,0202 & 0,1670 & 1,0000 & 1,00 & 47,75 & 13,65 & $-34,10$ \\
\hline & & & & 0,1980 & 0,64 & 6,05 & & 7,60 \\
\hline & & & & 0,0100 & 0,64 & 0,31 & & 13,34 \\
\hline \multirow[t]{3}{*}{ Gleissolo } & 14129 & 0,0132 & 0,1362 & 1,0000 & 1,00 & 25,41 & 14,14 & $-11,27$ \\
\hline & & & & 0,1980 & 0,64 & 3,22 & & 10,92 \\
\hline & & & & 0,0100 & 0,64 & 0,16 & & 13,98 \\
\hline \multirow[t]{3}{*}{ Latossolo } & 14129 & 0,0159 & 0,2735 & 1,0000 & 1,00 & 61,47 & 12,73 & $-48,74$ \\
\hline & & & & 0,1980 & 0,64 & 7,79 & & 4,94 \\
\hline & & & & 0,0100 & 0,64 & 0,39 & & 12,34 \\
\hline \multirow[t]{3}{*}{ Neossolo } & 14129 & 0,0192 & 0,2034 & 1,0000 & 1,00 & 55,27 & 10,48 & $-44,79$ \\
\hline & & & & 0,1980 & 0,64 & 7,00 & & 3,48 \\
\hline & & & & 0,0100 & 0,64 & 0,35 & & 10,13 \\
\hline \multirow[t]{3}{*}{ Planossolo } & 14129 & 0,0357 & 0,0690 & 1,0000 & 1,00 & 34,81 & 7,15 & $-27,66$ \\
\hline & & & & 0,1980 & 0,64 & 4,41 & & 2,74 \\
\hline & & & & 0,0100 & 0,64 & 0,22 & & 6,93 \\
\hline
\end{tabular}




\section{Conclusões}

A interferência antrópica pode condicionar os resultados obtidos pela aplicação da Equação Universal de Perdas de Solo. O mesmo solo apresenta perdas maiores ou menores em função da escolha do manejo e da pratica conservacionista. Do mesmo modo que o mesmo manejo e prática não resultam em perdas iguais se ossolos forem diferentes.

As perdas potenciais de solo foram maiores quando se considera o solo desnudo: 149,72 (Argissolo) a 25,41 $\mathrm{t} \mathrm{ha}^{-1} \mathrm{ano}^{-1}$ (Gleissolo), enquanto que, a pastagem combinada à semeadura em contorno promoveperdas de solo significativamente menores: 0,96 (Argissolos) a $0,16 \mathrm{t} \mathrm{ha}^{-1}$ ano $^{-1}$ (Gleissolos).

O uso de valores de tolerância em modelos de predição de perdas de solo permite buscar a combinação de fatores $\mathrm{C}$ e $\mathrm{P}$ que promovam uma perda de solos menor que o limite tolerável. Tal procedimento garante a sustentabilidade do sistema com potencial para a degradação.

\section{REFERÊNCIAS}

BERTOL, I.; ALMEIDA, J.A. Tolerância de perda de solo por erosão para os principais solos do Estado de Santa Catarina. Revista Brasileira de Ciência do Solo, Viçosa, v. 24. p. 657-668, 2000.

BERTONI, J. O espaçamento de terraços em culturas anuais, determinado em função das perdas por erosão. Bragantina, Campinas, SP, 18: 113-140 1959.

BERTONI, J.; LOMBARDI NETO, F.; BENATTI Jr., R. Equação de perdas de solo.Campinas: Instituto Agronômico, 1975. 23p. (Boletim Técnico, 21).

BERTONI, J.; LOMBARDI NETO, F.; Conservação do solo. $6^{\circ}$ edição. Piracicaba: Ícone, 2008. 356 p.

\section{CAMPOS, M.C.C. Pedogeomorfologia} aplicada a ambientes Amazônicos do médio rio madeira. Recife, 2009. 242p. Tese (Doutorado em Ciências do solo) Universidade Federal Rural de Pernambuco. Departamento de Agronomia.

CENAMO, M. C.; CARRERO, G. C.; SOARES, P. G. Redução de Emissões do Desmatamento e Degradação Florestal (REDD+): Estudo de Oportunidades para o
Sul do Amazonas. vol 1. Manaus, 2011. 56f. Série Relatórios Técnicos vol.1 Instituto de Conservação e Desenvolvimento Sustentável do Amazonas - IDESAM

DENARDIN, J.E. Erodibilidade do solo estimada por meio de parâmetros físicos e químicos. 1990. Tese (Doutorado) - Escola Superior de Agricultura Luiz de Queiroz, Universidade de São Paulo, Piracicaba, 1990.

EMPRESA BRASILEIRA DE PESQUISA AGROPECUÁRIA -EMBRAPA. Sistema Brasileiro de Classificação de Solos. Rio de Janeiro, Centro Nacional de Pesquisa de Solos, 2006. 306p.

GALINDO, I. C. L.; MARGOLIS, E. Tolerância de perdas por erosão para solos do estado de Pernambuco. Revista Brasileira de Ciência do Solo, Campinas, v. 13, p. 95-100, 1989.

GERARDO, K. A. R. F. Implementação da Equação Universal de Perdas de Solos (EUPS) em Sistema de Informações Geográficas. São José do Campos. Disponível em:

<gerardokuntschik.tripod.com/Papers/cbcs.pd f>. Acesso em 15 de outubro de 2011. 
LIMA, E. R. V. Erosão do solo: fatores condicionantes e modelagem matemática.

Logepa, ano 1, n 1, 2003.

MARTINS FILHO, M. V. Equação Universal de Perdas de Solo. Unesp, Jaboticabal, 2004. $47 \mathrm{p}$.

LOMBARDI NETO, F.; BERTONI, J. Tolerância de perdas de terras para solos do Estado de São Paulo. Campinas: Instituto Agronômico, 1975. 12p. (Boletim Técnico, 28).

OLIVEIRA, F. P. DE.; Determinação da tolerância de perdas por erosão para as principais Ordens de solos do Estado da Paraíba. Areia, 2004. 89f. Trabalho de conclusão de curso (Graduação em Engenharia Agronômica) - Universidade Federal da Paraíba, Centro de Ciências Agrárias.

ROOSE, E. J. Application of the universal soil loss equation of Wischmeier and Smith in West Africa. In: GREENLAND, D. J.; LAL, R. (Ed.) Soil conservation and management in the humid tropics. Chichester: Wiley, 1977. p. 177-187.

SMITH, R. M.; STAMEY, W. L.

Determining the range of tolerable erosion. Soil Science, Baltimores, v. 100, p.414-424, 1965.

VIEIRA, M. N. F.; VIEIRA, L. S. Tolerância de perdas de terras para alguns solos amazônicos. Boletim da FCAP, Belém, n. 19, p. 71-77, 1990.

WISCHMEIER, W.H; SMITH, D.D. Predicting rainfall erosion losses: A guide to conservation planning. Washington: USDA, 1978. 58p. (Agricultural Handbook, 53). 